\title{
Limits on the spatial variations of the electron-to-proton mass ratio in the Galactic plane ${ }^{\star}$
}

\author{
S. A. Levshakov ${ }^{1,2,3}$, D. Reimers ${ }^{1}$, C. Henkel ${ }^{4,5}$, B. Winkel ${ }^{4}$, A. Mignano ${ }^{6}$, M. Centurión ${ }^{7}$, and P. Molaro ${ }^{7,8}$ \\ ${ }^{1}$ Hamburger Sternwarte, Universität Hamburg, Gojenbergsweg 112, 21029 Hamburg, Germany \\ e-mail: lev@astro.ioffe.rssi.ru \\ 2 Ioffe Physical-Technical Institute, Polytekhnicheskaya Str. 26, 194021 St. Petersburg, Russia \\ 3 St. Petersburg Electrotechnical University "LETI", Prof. Popov Str. 5, 197376 St. Petersburg, Russia \\ ${ }^{4}$ Max-Planck-Institut für Radioastronomie, Auf dem Hügel 69, 53121 Bonn, Germany \\ 5 Astronomy Department, King Abdulaziz University, PO Box 80203, 21589 Jeddah, Saudi Arabia \\ ${ }^{6}$ INAF - Istituto di Radio Astronomia, via P. Gobetti 101, Bologna, Italy \\ 7 INAF - Osservatorio Astronomico di Trieste, via Tiepolo 11, 34131 Trieste, Italy \\ 8 Centro de Astrofsica, Universidade do Porto, Rua das Estrelas, 4150-762 Porto, Portugal
}

Received 26 August 2013 / Accepted 7 October 2013

\section{ABSTRACT}

\begin{abstract}
Aims. We aim to validate the Einstein equivalence principle (local position invariance) by limiting the fractional changes in the electron-to-proton mass ratio, $\mu=m_{\mathrm{e}} / m_{\mathrm{p}}$, measured in Galactic plane objects.

Methods. High-resolution spectral observations of dark clouds in the inversion line of $\mathrm{NH}_{3}(1,1)$ and pure rotational lines of other molecules (the so-called ammonia method) were performed at the Medicina 32-m and the Effelsberg 100-m radio telescopes to measure the radial velocity offsets, $\Delta R V=V_{\text {rot }}-V_{\text {inv }}$, between the rotational and inversion transitions, which have different sensitivities to the value of $\mu$.

Results. In our previous observations (2008-2010), a mean offset of $\langle\Delta R V\rangle=0.027 \pm 0.010 \mathrm{~km} \mathrm{~s}^{-1}(3 \sigma$ confidence level (C.L.)) was measured. To test for possible hidden errors, we carried out additional observations of a sample of molecular cores in 2010-2013. As a result, a systematic error with an amplitude $\sim 0.02 \mathrm{~km} \mathrm{~s}^{-1}$ in the radial velocities was revealed. The averaged offset between the radial velocities of the rotational transitions of $\mathrm{HC}_{3} \mathrm{~N}(2-1), \mathrm{HC}_{5} \mathrm{~N}(9-8), \mathrm{HC}_{7} \mathrm{~N}(16-15), \mathrm{HC}{ }_{7} \mathrm{~N}(21-20)$, and $\mathrm{HC}_{7} \mathrm{~N}(23-22)$, and the inversion transition of $\mathrm{NH}_{3}(1,1)$ is $\langle\Delta R V\rangle=0.003 \pm 0.018 \mathrm{~km} \mathrm{~s}^{-1}\left(3 \sigma\right.$ C.L.). This value, when interpreted in terms of $\Delta \mu / \mu=\left(\mu_{\mathrm{obs}}-\mu_{\mathrm{lab}}\right) / \mu_{\mathrm{lab}}$,

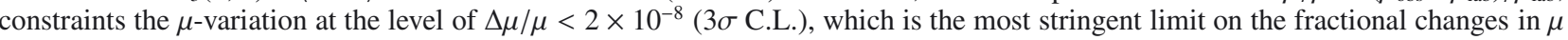
based on astronomical observations.
\end{abstract}

Key words. line: profiles - ISM: molecules - radio lines: ISM - techniques: radial velocities - elementary particles

\section{Introduction}

This study is aimed at testing whether the dimensionless physical constant, the electron-to-proton mass ratio, $\mu=m_{\mathrm{e}} / m_{\mathrm{p}}$, is really constant, or whether it varies with space and time. The latter would imply, at some level, a violation of the Einstein equivalence principle (EEP), that is, local position invariance (LPI) and local Lorentz invariance (LLI), as suggested in a number of unification theories (for reviews, see, e.g., Uzan 2011; Liberati 2013). In particular, a changing fine-structure constant, $\alpha=e^{2} / \hbar c$, accompanied by variation in other coupling constants can be associated with a violation of LLI (Kostelecký et al. 2003). LPI, on the other hand, postulates that the outcome of any local nongravitational experiment is independent of where and when it is performed, that is, that the fundamental physical laws are space-time invariant.

Experimental validation of EEP is, therefore, one of the pillars of the Standard Model (SM) of particle physics, allowing us to probe the applicability limits of the SM in testing the most accurate theories, such as quantum electrodynamics and/or in

\footnotetext{
* Based on observations obtained with the Effelsberg 100-m telescope operated by the Max-Planck Institut für Radioastronomie on behalf of the Max-Planck-Gesellschaft (Germany), and with the Medicina 32-m telescope operated by INAF (Italy).
}

searching for new types of interactions. At the same time, precision limits delivered from these experiments serve as restrictions for the numerous new theories beyond the SM and can help to distinguish between them.

Two coupling constants in question are of particular interest for astrophysical experiments since their fractional changes $\Delta \mu / \mu=\left(\mu_{\mathrm{obs}}-\mu_{\mathrm{lab}}\right) / \mu_{\mathrm{lab}}$, and $\Delta \alpha / \alpha=\left(\alpha_{\mathrm{obs}}-\alpha_{\mathrm{lab}}\right) / \alpha_{\mathrm{lab}}$ can be measured accurately from spectra of Galactic and extragalactic sources. Because the fine-structure constant sets the scale of the electromagnetic interaction, experiments in which electrons interact with electromagnetic fields (e.g., forming atomic and molecular spectra in this way) can be used to determine $\alpha$. The electron-to-proton mass ratio is known to define the vibrational $\left(E_{\mathrm{vib}} \propto \sqrt{\mu}\right)$ and rotational $\left(E_{\mathrm{rot}} \propto \mu\right)$ frequencies of molecular spectra. On the other hand, the electron mass $m_{\mathrm{e}}$ is related to the vacuum expectation value of the Higgs field (i.e., the scale of the weak nuclear force), and $m_{\mathrm{p}}$ - to the quantum chromodynamics energy scale (i.e., the strong nuclear force). Therefore, molecular transitions can be used to determine $\mu$ and thus the ratio between weak and strong nuclear forces.

In spite of some claims of marginal detections of changes in either $\alpha$ or $\mu$ at high redshifts, none have been confirmed through independent observations. As shown in Fig. 1, the most precise constraints on $\Delta \alpha / \alpha$ and $\Delta \mu / \mu$ based on astronomical spectra 


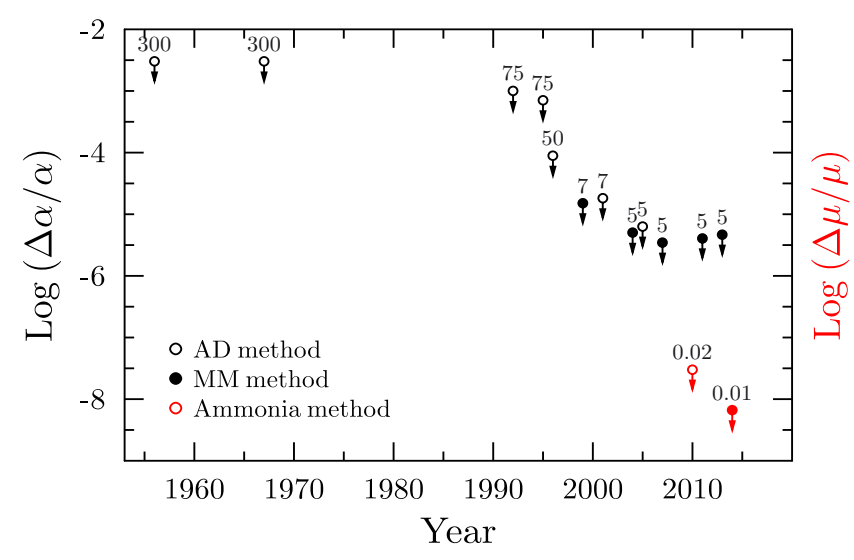

Fig. 1. Astronomical constraints on $\alpha$ - and $\mu$-variations ( $1 \sigma$ C.L.) for the period from 1956 to 2013. Above each point, the spectral resolution (FWHM in $\mathrm{km} \mathrm{s}^{-1}$ ) is indicated. $\alpha$-variation constraints are based on the alkali-doublet (AD) and many-multiplet (MM) methods applied to extragalactic targets, whereas the $\mu$-variation is based on the ammonia method (Galactic targets). The data points for the AD method are taken from Savedoff (1956), Bahcall et al. (1967), Levshakov (1992), Varshalovich \& Potekhin (1995), Varshalovich et al. (1996), Murphy et al. (2001), and Chand et al. (2005); for the MM method they are taken from Webb et al. (1999), Quast et al. (2004), Srianand et al. (2007), Agafonova et al. (2011), and Molaro et al. (2013); for the ammonia method they are taken from Paper II (red circle), and the current value (red dot). The figure shows that limits on the $\alpha$ - and $\mu$-variations just follow the spectral resolution approximately as $\Delta \alpha / \alpha$ (or $\Delta \mu / \mu) \propto 1 / 10$ th of the pixel size.

just follow the available spectral resolution. Up to now, no signals have yet been detected in the range of fractional changes from $\sim 3 \times 10^{-2}$ to $\sim 3 \times 10^{-8}$. Thus, any progress in improving the existing limits can be achieved from observations of narrow spectral lines involving higher spectral resolutions to resolve their profiles completely. At the moment, the resolution of radio telescopes exceeds that of optical facilities by order(s) of magnitude; an additional and very attractive property of microwave radio observations is that some molecular transitions from this frequency range are extremely sensitive to the putative variations of the fundamental physical constants (for a review see, e.g., Kozlov \& Levshakov 2013).

In 2007, Flambaum \& Kozlov proposed the so-called ammonia method to test the variability of $\mu$. It compares the relative shifts between the inversion transition of $\mathrm{NH}_{3}(1,1)$ and pure rotational transitions of other molecules tracing closely the ammonia spatial distribution. The sensitivity coefficient, $Q_{\text {inv }}$, of the inversion transition to the $\mu$-variation is 4.46 times higher than that of the rotational transition $\left(Q_{\mathrm{rot}}=1\right)$. Using this method for a sample of cold molecular cores from the Galactic plane, we obtained the following estimates of the spatial $\mu$-variations: $\Delta \mu / \mu=$ $\left(1.3 \pm 0.8_{\text {stat }} \pm 0.3_{\text {sys }}\right) \times 10^{-8}[1 \sigma$ confidence level (C.L.) $]$ with the Medicina 32-m telescope (Levshakov et al. 2010a, Paper I), and $\Delta \mu / \mu=\left(2.6 \pm 0.1_{\text {stat }} \pm 0.3_{\text {sys }}\right) \times 10^{-8}$, based on observations with the Effelsberg 100-m telescope (Levshakov et al. 2010b, Paper II). The given systematic errors are dominated by uncertainties of the rest frame frequencies of the observed molecular transitions. However, these measurements have been carried out at the highest sensitivity available (maximum performance) and, thus, the true systematic error can be in fact larger because of potentially inherent instrumental errors. A quantitative assessment of this uncertainty is not a straightforward procedure and requires in general both a special design of measurements and a cross-checking of results obtained with different instruments.
To provide such an assessment we repeated observations of the same targets, employing spectrometers with spectral resolutions, which are different from those previously used. Additionally, we performed a set of measurements at the Effelsberg telescope to obtain a more detailed characteristic of instrumental instabilities that may affect the values of line radial velocities. Here we present the results.

\section{Observations}

The following molecular transitions were observed towards cold $\left(T_{\text {kin }} \sim 10 \mathrm{~K}\right)$ and dense $\left(n_{\mathrm{H}_{2}} \sim 10^{4} \mathrm{~cm}^{-3}\right)$ starless molecular cores from the Galactic plane listed in Table 1: $\mathrm{NH}_{3}(J, K)=$ $(1,1) 23.7 \mathrm{GHz}, \mathrm{HC}_{3} \mathrm{~N}(J=2-1) 18.2 \mathrm{GHz}, \mathrm{HC}_{5} \mathrm{~N}(J=9-8)$ $23.9 \mathrm{GHz}, \mathrm{HC}_{7} \mathrm{~N}(J=16-15) 18.0 \mathrm{GHz}, \mathrm{HC}_{7} \mathrm{~N}(J=21-20)$ $23.7 \mathrm{GHz}$, and $\mathrm{HC}_{7} \mathrm{~N}(J=23-22) 25.9 \mathrm{GHz}$. We used the Medicina $32-\mathrm{m}$ and the Effelsberg 100-m radio telescope as described in Papers I and II, and in Levshakov et al. (2013).

In November 2010, cores L1512 and L1498 were reobserved with the 32-m Medicina telescope using the highresolution digital spectrometer $\mathrm{MSpec0}$ with a channel separation of $0.494 \mathrm{kHz}$ (8192 channels). This corresponds to $\Delta_{\mathrm{ch}}=0.006 \mathrm{~km} \mathrm{~s}^{-1}$ at the position of the ammonia inversion transition $\mathrm{NH}_{3}(1,1)$. For comparison, observations of core L1512 in 2008 with the digital spectrometer ARCOS (ARcetri COrrelation Spectrometer) had $\Delta_{\mathrm{ch}}=0.062 \mathrm{~km} \mathrm{~s}^{-1}$ (Paper I). The angular resolutions at the frequencies of the $\mathrm{NH}_{3}(1,1)$ and $\mathrm{HC}_{3} \mathrm{~N}(2-1)$ lines were $1.6^{\prime}$ and $2.1^{\prime}$, respectively. The observations were performed in position-switching mode (PSW). The rms pointing uncertainty was $\lesssim 25^{\prime \prime}$.

Observations in 2011-2013 with the Effelsberg 100-m telescope targeted a sample of nine cores from Table 1 . The source coordinates were taken from Paper I and Levshakov et al. (2013), except for L1498A, for which they were estimated from Fig. 3 in Kuiper et al. (1996). The source L1498A is a gas condensation within the L1498 dark cloud separated by $2^{\prime}$ from the $\mathrm{NH}_{3}$ reference position.

In 2011, the measurements were obtained in the frequencyswitching (FSW) mode using a frequency throw of $\pm 2.5 \mathrm{MHz}$. The backend was a fast Fourier-transform spectrometer (FFTS), operated with a bandwidth of $20 \mathrm{MHz}$, which simultaneously provided 16384 channels for each polarization. The resulting channel width was $\Delta_{\mathrm{ch}}=0.015 \mathrm{~km} \mathrm{~s}^{-1}$. However, the true velocity resolution is about 1.6 times coarser.

In 2012-2013, we performed the measurements in the PSW mode with the backend XFFTS (eXtended bandwidth FFTS) operating with $100 \mathrm{MHz}$ bandwidth and providing 32768 channels for each polarization. The resulting channel width was $\Delta_{\mathrm{ch}}=0.039 \mathrm{~km} \mathrm{~s}^{-1}$, but the true velocity resolution is 1.16 times lower (Klein et al. 2012).

\section{Results}

The spatial distribution of the molecular cores in Galactic coordinates are shown in Fig. 2. The cores are located towards both the Galactic center and anti-center, at distances $100 \mathrm{pc}$ $\lesssim D \lesssim 300$ pc from the Sun, and close to the Galactic equator, $-19^{\circ}<b<15^{\circ}$. The selected sources are known to have narrow molecular emission lines (full width at half maximum, $F W H M<1 \mathrm{~km} \mathrm{~s}^{-1}$ ), which makes them the most suitable targets for precise measurements of relative radial velocities (RV). At first, we consider the results obtained at Effelsberg, then those at Medicina. 
Table 1. Source positions and radial velocities.

\begin{tabular}{|c|c|c|c|c|c|c|}
\hline \multirow[t]{2}{*}{ No. } & \multirow[t]{2}{*}{ Core } & \multicolumn{2}{|c|}{ Position } & \multirow{2}{*}{$\begin{array}{c}V_{\mathrm{LSR}} \\
\left(\mathrm{km} \mathrm{s}^{-1}\right)\end{array}$} & \multirow[t]{2}{*}{ Other name } & \multirow{2}{*}{$\begin{array}{l}\text { Telescope } \\
\text { used }\end{array}$} \\
\hline & & $\begin{array}{c}\alpha_{2000} \\
\left(\mathrm{~h}: \mathrm{m}:{ }^{\mathrm{s}}\right)\end{array}$ & $\begin{array}{c}\delta_{2000} \\
\left({ }^{\circ}:^{\prime}: "\right)\end{array}$ & & & \\
\hline 1 & L1498 & $04: 10: 51$ & $+25: 09: 58$ & 7.81 & & $32-\mathrm{m} / 100-\mathrm{m}$ \\
\hline 2 & L1498A & $04: 10: 58$ & $+25: 09: 15$ & 7.75 & & $100-\mathrm{m}$ \\
\hline 3 & Do279P6 & 18:29:07 & $+00: 30: 51$ & 7.75 & Ser G3-G6 ${ }^{a}$ & $100-\mathrm{m}$ \\
\hline 4 & Do279P12 & $18: 29: 47$ & $+01: 14: 53$ & 7.59 & BDN $31.57+5.37^{b}$ & $100-\mathrm{m}$ \\
\hline 5 & SS3 & $18: 29: 57$ & $-02: 00: 10$ & 7.39 & & $100-\mathrm{m}$ \\
\hline 6 & Do279P8 & 18:30:04 & $-02: 48: 14$ & 8.52 & & $100-\mathrm{m}$ \\
\hline 7 & $\mathrm{Ka} 05$ & $19: 37: 01$ & $+07: 34: 30$ & 8.40 & $\mathrm{~B} 335^{c}, \mathrm{CB} 199^{d}$ & $100-\mathrm{m}$ \\
\hline 8 & L1251C & $22: 35: 54$ & $+75: 18: 55$ & -4.73 & & $100-\mathrm{m}$ \\
\hline 9 & $\mathrm{~L} 1512$ & $05: 04: 10$ & $+32: 43: 09$ & 7.11 & & $32-\mathrm{m} / 100-\mathrm{m}$ \\
\hline
\end{tabular}

References. (a) Cohen \& Kuhi (1979); (b) Dobashi et al. (2005); (c) Barnard (1927); (d) Clemens and Barvainis (1988).

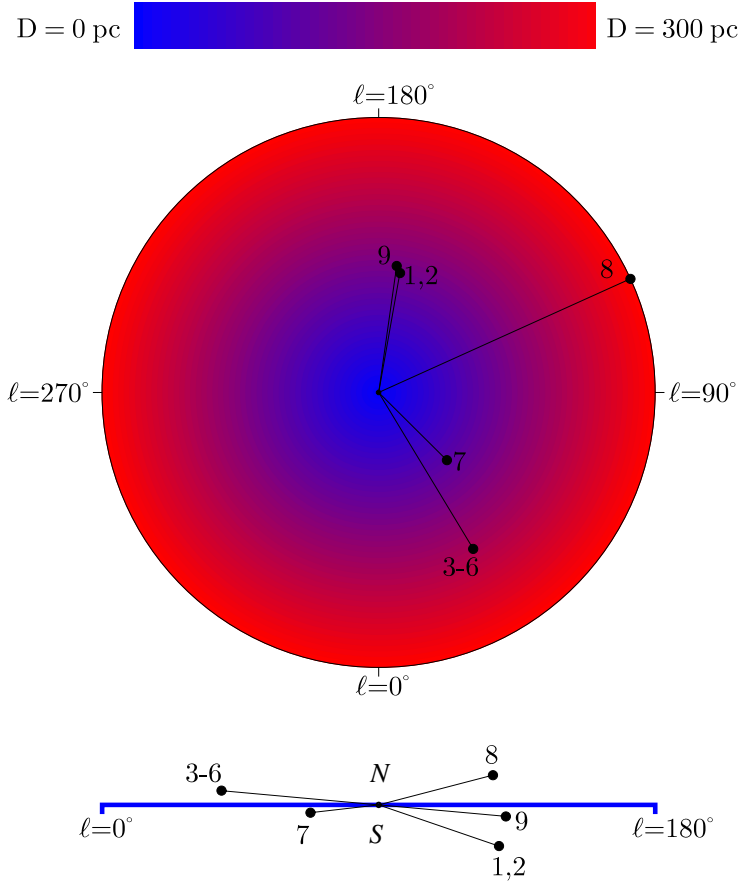

Fig. 2. Schematic location of the observed molecular cores in projection onto the Galactic plane and equator. The Galactic center longitude is $\ell=0^{\circ}$, by definition. The distance scale is given by the wedge at the top of the figure. Numbers mark sources from Table 1.

\subsection{Effelsberg observations}

To verify the reproducibility of the relative $\mathrm{RVs}$ of the $\mathrm{NH}_{3}(1,1)$ and $\mathrm{HC}_{3} \mathrm{~N}(2-1)$ lines we re-observed the two molecular cores L1512 and L1498 in 2011. The procedure was the same as during the 2010 observations, that is, cores were mapped at the same offsets and in the same lines: in the $(1,1)$ inversion transition of $\mathrm{NH}_{3}$ complemented by rotational lines of other molecular species.

Line parameters such as the total optical depth in the transition, $\tau$, the radial velocity, $V_{\mathrm{LSR}}$, the linewidth, $\Delta v$, and the amplitude, $\mathcal{A}$, were estimated by fitting a one-component Gaussian model to the observed spectra. The model was defined by Eqs. ((8)-(10)) in Paper I. Since we are mostly interested in the model parameters $V_{\mathrm{LSR}}$ and $\Delta v$, their values are listed in Table 2. Given in parentheses are the $1 \sigma$ errors of $V_{\mathrm{LSR}}$ and, directly below, $\Delta v$ obtained from the diagonal elements of the covariance matrix calculated for the minimum of $\chi^{2}$. The last column presents the differences $\Delta R V=V_{\text {rot }}-V_{\text {inv }}$ between the radial velocities of the rotational lines and the inversion $(1,1)$ line of $\mathrm{NH}_{3}$, as well as their $1 \sigma$ errors (numbers in parentheses). The offsets $\Delta R V$ provide a sensitive limit to the variation of $\mu$ (Flambaum \& Kozlov 2007):

$\Delta \mu / \mu=\left(V_{\mathrm{rot}}-V_{\mathrm{inv}}\right) /\left[\left(Q_{\mathrm{inv}}-Q_{\mathrm{rot}}\right) c\right] \approx 0.3 \Delta V / c$,

where $c$ is the speed of light, and $Q_{\text {inv }}, Q_{\text {rot }}$ are the corresponding sensitivity coefficients to changes in $\mu$.

The RVs measured in 2010 (Paper II) and 2011 at different radial distances along the main diagonal cuts towards L1512 and L1498 are shown in Fig. 3. The velocity offsets $\Delta R V$ exhibit quite a different behavior in these two years: at the same positions the changes of $\Delta R V$ considerably exceed its uncertainty interval ( $\lesssim 0.003 \mathrm{~km} \mathrm{~s}^{-1}$, see Figs. 3 and 4 in Paper II), which is probably caused by unknown systematic errors.

To determine the source of these errors, we performed in 2012 a set of continuous observations of L1512 and L1498 targeting their ammonia peaks. Observing in PSW mode, we also used different OFF positions (for L1512) to search for possible contamination from a putative extended background ammonia emission (which was not detected). The resulting time series are shown in Fig. 4. The exposure time of each subscan was $150 \mathrm{~s}$. The RV values fluctuate with an amplitude of $\approx \pm 0.01 \mathrm{~km} \mathrm{~s}^{-1}$, that is, $\approx 1 / 4$ th of the channel width.

In our observations, the sky frequencies were reset at the onset of each subscan. Therefore, the longer a subscan, the higher the error caused by Doppler shifts during the exposure time (e.g., for a $5 \mathrm{~min}$ scan, it is about $0.004 \mathrm{~km} \mathrm{~s}^{-1}$ at Effelsberg latitude). We corrected some of our observations to account for residual Doppler shifts. This did not lead to a significant change in the results, however.

To determine whether the sky frequency is identical with the frequency coming out of the backend we carried out a test with an artificial signal at $22000.78125 \mathrm{MHz}$. The synthesizer frequency was accurate to about $1 \mathrm{~Hz}$, and the frequency scale was found to be accurate to about $32 \mathrm{~Hz}\left(\approx 0.0004 \mathrm{~km} \mathrm{~s}^{-1}\right)$.

Another source of errors that can affect the $\Delta \mu / \mu$ estimates with the ammonia method is the possible segregation of molecules in molecular cores, which gives rise to systematic shifts of RVs. Figure 5 shows the $\Delta R V$ offsets measured in 2013 (filled circles) towards eight molecular cores at different positions indicated in Col. 2 of Table 2 and Cols. 3 and 4 of Table 1. Previous $\Delta R V$ values, obtained in 2009-2011, are marked by open symbols. A spread of the velocity offsets $\Delta R V$ is clearly seen. 
Table 2. Radial velocities, $V_{\mathrm{LSR}}$, linewidths (FWHM), $\Delta v$, and velocity offsets, $\Delta R V=V_{\text {rot }}-V_{\text {inv }}$, measured with the Effelsberg $100-\mathrm{m}$ and the Medicina 32-m telescopes.

\begin{tabular}{|c|c|c|c|c|c|c|c|c|}
\hline Source & $\begin{array}{l}\text { Offset } \\
\Delta \alpha, \Delta \delta \\
\left({ }^{\prime \prime}\right),\left({ }^{\prime \prime}\right)\end{array}$ & $\mathrm{NH}_{3}(1,1)$ & $\mathrm{HC}_{7} \mathrm{~N}(23-22)$ & $\begin{array}{c}V_{\mathrm{LSR}}, \mathrm{km} \mathrm{s}^{-1} \\
\mathrm{HC}_{7} \mathrm{~N}(16-15)\end{array}$ & $\begin{array}{l}\Delta v, \mathrm{~km} \mathrm{~s}^{-1} \\
\mathrm{HC}_{7} \mathrm{~N}(21-20)\end{array}$ & $\mathrm{HC}_{5} \mathrm{~N}(9-8)$ & $\mathrm{HC}_{3} \mathrm{~N}(2-1)$ & $\begin{array}{c}\Delta \mathrm{RV} \\
\mathrm{km} \mathrm{s}^{-1}\end{array}$ \\
\hline \multicolumn{9}{|l|}{ Effelsberg: } \\
\hline \multirow[t]{2}{*}{ L1498A } & \multirow[t]{2}{*}{0,0} & \multirow{2}{*}{$\begin{array}{l}7.753(1)^{a} \\
0.204(3)\end{array}$} & \multirow[t]{2}{*}{$\begin{array}{l}7.760(2)^{a} \\
0.096(7)\end{array}$} & & $\ldots$ & $\ldots$ & $\ldots$ & $0.007(2)$ \\
\hline & & & & $\begin{array}{l}7.753(6)^{a} \\
0.13(1)\end{array}$ & $\ldots$ & $\ldots$ & $\ldots$ & $0.000(6)$ \\
\hline L1498 & 0,0 & $\begin{array}{l}7.798(2)^{a} \\
0.186(5)^{2}\end{array}$ & $\ldots$ & $\ldots$ & $\begin{array}{l}7.78(1)^{a} \\
0.08(2)\end{array}$ & $\ldots$ & $\ldots$ & $-0.02(1)$ \\
\hline \multirow[t]{2}{*}{ Do279P6 } & 40,40 & $\begin{array}{l}8.045(7)^{c} \\
0.63(1)\end{array}$ & $\ldots$ & $\ldots$ & $\ldots$ & $\begin{array}{l}8.00(6)^{a} \\
0.04(3)\end{array}$ & $\ldots$ & $-0.05(6)$ \\
\hline & $-160,-80$ & $\begin{array}{l}7.69(1)^{c} \\
0.96(3)\end{array}$ & $\ldots$ & $\ldots$ & $\ldots$ & $\begin{array}{l}7.77(4)^{a} \\
0.5(1)\end{array}$ & $\ldots$ & $0.08(4)$ \\
\hline Do279P8 & 0,0 & $\begin{array}{l}8.524(7)^{c} \\
0.26(2)\end{array}$ & $\cdots$ & $\cdots$ & $\cdots$ & $\begin{array}{l}8.75(1)^{a} \\
0.13(2)\end{array}$ & $\ldots$ & $0.23(1)$ \\
\hline Do279P12 & 40,40 & $\begin{array}{l}8.290(8)^{c} \\
0.91(2)\end{array}$ & $\ldots$ & $\ldots$ & $\ldots$ & $\begin{array}{l}8.17(3)^{a} \\
0.23(5)\end{array}$ & $\ldots$ & $-0.12(3)$ \\
\hline $\mathrm{Ka} 05$ & 0,0 & $\begin{array}{l}8.401(4)^{b} \\
0.365(8)\end{array}$ & $\ldots$ & $\ldots$ & $\ldots$ & $\begin{array}{l}8.38(1)^{a} \\
0.32(8)\end{array}$ & $\ldots$ & $-0.02(1)$ \\
\hline \multirow[t]{2}{*}{ SS3 } & 0,160 & $\begin{array}{l}7.622(6)^{b} \\
0.396(3)\end{array}$ & $\ldots$ & $\ldots$ & $\ldots$ & $\begin{array}{l}7.65(2)^{a} \\
0.50(3)\end{array}$ & $\ldots$ & $0.03(2)$ \\
\hline & 0,80 & $\begin{array}{l}7.668(2)^{b} \\
0.490(5)\end{array}$ & $\cdots$ & $\ldots$ & $\cdots$ & $\begin{array}{l}7.53(1)^{a} \\
0.38(3)\end{array}$ & $\ldots$ & $-0.14(1)$ \\
\hline \multirow[t]{6}{*}{ L1251C } & 0,0 & $\begin{array}{l}-4.722(3)^{a} \\
0.290(7)\end{array}$ & $\ldots$ & $\ldots$ & $\ldots$ & $\begin{array}{l}-4.64(2)^{a} \\
0.33(3)\end{array}$ & $\ldots$ & $0.08(2)$ \\
\hline & 0,40 & $\begin{array}{l}-4.616(9)^{a} \\
0.30(2)\end{array}$ & $\cdots$ & $\ldots$ & $\cdots$ & $\begin{array}{l}-4.59(2)^{a} \\
0.28(3)\end{array}$ & $\ldots$ & $0.03(2)$ \\
\hline & 40,0 & $\begin{array}{l}-4.733(5)^{a} \\
0.28(1)\end{array}$ & $\ldots$ & $\ldots$ & $\ldots$ & $\begin{array}{l}-4.67(1)^{a} \\
0.27(3)\end{array}$ & $\ldots$ & $0.06(1)$ \\
\hline & $-40,0$ & $\begin{array}{l}-4.718(6)^{a} \\
0.29(1)\end{array}$ & $\ldots$ & $\ldots$ & $\ldots$ & $\begin{array}{l}-4.69(2)^{a} \\
0.30(8)\end{array}$ & $\ldots$ & $0.03(2)$ \\
\hline & 80,0 & $\begin{array}{l}-4.755(6)^{a} \\
0.29(1)\end{array}$ & $\ldots$ & $\ldots$ & $\ldots$ & $\begin{array}{l}-4.70(4)^{a} \\
0.37(7)\end{array}$ & $\ldots$ & $0.06(4)$ \\
\hline & $-40,-40$ & $\begin{array}{l}-4.805(6)^{a} \\
0.35(1)\end{array}$ & $\ldots$ & $\ldots$ & $\ldots$ & $\begin{array}{l}-4.89(6)^{a} \\
0.28(2)\end{array}$ & $\ldots$ & $-0.09(6)$ \\
\hline \multicolumn{9}{|l|}{ Medicina: } \\
\hline L1512 & 0,0 & $\begin{array}{l}7.160(2)^{d} \\
0.191(7)\end{array}$ & $\ldots$ & $\ldots$ & $\ldots$ & $\ldots$ & $\begin{array}{l}7.161(2)^{d} \\
0.16(1)\end{array}$ & $0.001(3)$ \\
\hline L1498 & 0,0 & $\begin{array}{l}7.883(3)^{d} \\
0.185(8)\end{array}$ & $\ldots$ & $\ldots$ & $\ldots$ & $\ldots$ & $\begin{array}{l}7.882(2)^{d} \\
0.16(1)\end{array}$ & $-0.001(4)$ \\
\hline
\end{tabular}

Notes. Observations: ${ }^{(a)} 2013,{ }^{(b)} 2012,{ }^{(c)} 2011,{ }^{(d)}$ 2010. Rest frame frequencies: $\mathrm{NH}_{3}(1,1) 23694.495487(60) \mathrm{MHz}, \mathrm{HC} 7 \mathrm{~N}(23-22)$ 25 943.8549(7) MHz, HC ${ }_{7} \mathrm{~N}(21-20)$ 23687.8974(6) $\mathrm{MHz}, \mathrm{HC}_{7} \mathrm{~N}(16-15) 18047.9697(5) \mathrm{MHz}, \mathrm{HC}_{5} \mathrm{~N}(9-8) 23$ 963.9007(1) $\mathrm{MHz}, \mathrm{HC}_{3} \mathrm{~N}(2-1)$ 18 196.2169(2) MHz. Notations: upper values are $V_{\mathrm{LSR}}$, lower values $-\Delta v$; the values in parenthesis give $1 \sigma$ standard deviations, referring to the last given digit of the respective parameter.

Thus, we conclude that noise in the $\Delta R V$ values consists of at least two components. One is due to chemical differentiation and velocity gradients within the molecular cores, possibly amplified by small variations in the telescope pointing. The other may originate from the different optical depths of the hyperfine structure transitions, which may lead to some scatter in the RVs. However, all these effects may act in opposite directions from one observation to another, and, because they are averaged over a sample of targets, should be reduced to some extent. Applied to our sample shown in Fig. 5 ( $n=19$ independent offsets $\Delta R V$ ), this gives a weighted mean $\langle\Delta R V\rangle=0.003 \pm 0.018 \mathrm{~km} \mathrm{~s}^{-1}(3 \sigma$ C.L.). When interpreted in terms of $\Delta \mu / \mu=\left(\mu_{\mathrm{obs}}-\mu_{\mathrm{lab}}\right) / \mu_{\mathrm{lab}}$, this value of $\langle\Delta R V\rangle$ constrains the $\mu$-variation at the level of $\Delta \mu / \mu<$ $2 \times 10^{-8}$ ( $3 \sigma$ C.L.), which is the most stringent limit on the spatial variation of $\mu$ based on radio astronomical observations.

We note in passing that mapping of the dense molecular cores in different molecular lines shows that there is, in general, a good correlation between the $\mathrm{NH}_{3}, \mathrm{~N}_{2} \mathrm{H}^{+}$, and $\mathrm{HC}_{3} \mathrm{~N}$ distributions (Fuller \& Myers 1993; Hotzel et al. 2004; Tafalla et al. 2004; Pagani et al. 2009). However, in some clouds $\mathrm{NH}_{3}$ is not traced by $\mathrm{HC}_{3} \mathrm{~N}$, for instance, in the dark cloud TMC-1, where peaks of emission lines are offset by $7^{\prime}$ (Olano et al. 1988). In our case, we observe systematic velocity shifts between $\mathrm{NH}_{3}$ and other species. This is expected since C-bearing molecules are usually distributed in the outer layers of the cores rich in depletion-sensitive molecules, whereas N-bearing molecules, which are depletion-resistant species, trace the inner cores (examples are given in, e.g., Tafalla et al. 2006).

\subsection{Medicina observations}

The $\mathrm{NH}_{3}(1,1)$ and $\mathrm{HC}_{3} \mathrm{~N}(2-1)$ lines towards the cores $\mathrm{L} 1512$ and L1498 are among the narrowest molecular lines known in the interstellar medium. The observed FWHM linewidths of the completely resolved hyperfine components, the $F_{1}^{\prime} \rightarrow$ $F_{1}, F^{\prime} \rightarrow F=0 \rightarrow 1,1 / 2 \rightarrow 3 / 2$ line of $\mathrm{NH}_{3}(1,1)$ and the 
S. A. Levshakov et al.: Limits on the spatial variations of the electron-to-proton mass ratio in the Galactic plane

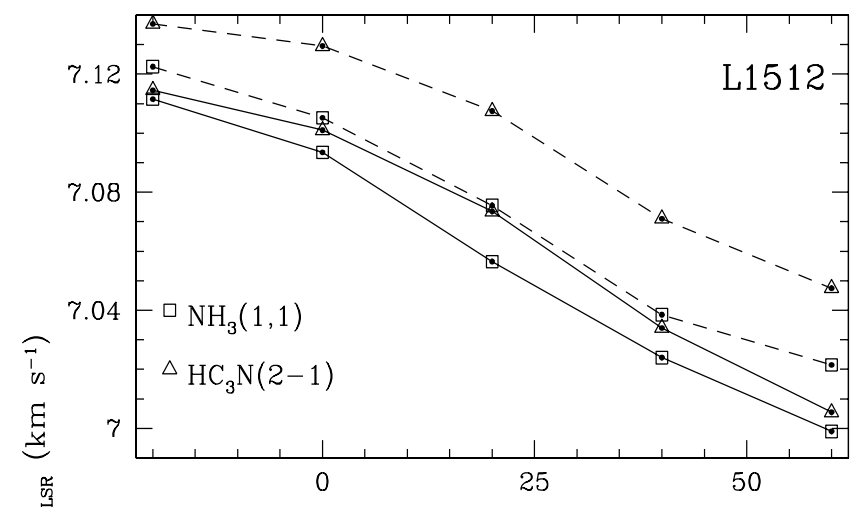

$>^{\frac{2}{3}}$

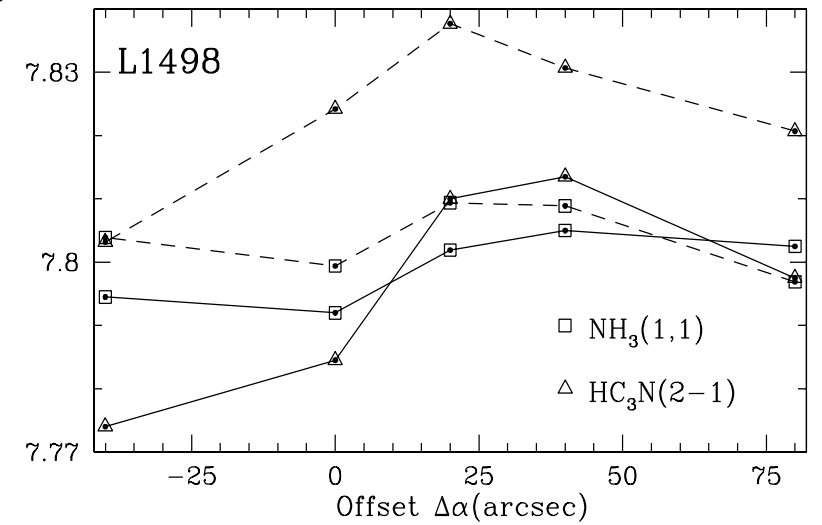

Fig. 3. Line-of-sight velocities $\left(V_{\mathrm{LSR}}\right)$ of $\mathrm{NH}_{3}(J, K)=(1,1)$ (squares) and $\mathrm{HC}_{3} \mathrm{~N} J=2-1$ (triangles) along the main diagonal cuts towards the molecular cores L1512 and L1498 measured in 2010 (dashed lines) and in 2011 (solid lines) at the Effelsberg 100-m radio telescope. The halfpower beam width at $23 \mathrm{GHz}$ is $40^{\prime \prime}\left(50^{\prime \prime}\right.$ at $\left.18 \mathrm{GHz}\right)$, the backend is the fast Fourier-transform spectrometer (FFTS) with a channel separation $\Delta_{\mathrm{ch}}=0.015 \mathrm{~km} \mathrm{~s}^{-1}\left(0.020 \mathrm{~km} \mathrm{~s}^{-1}\right.$ at $\left.18 \mathrm{GHz}\right)$.

$F^{\prime} \rightarrow F=3 \rightarrow 2$ transition of $\mathrm{HC}_{3} \mathrm{~N}(2-1)$, are $\Delta v=0.19 \mathrm{~km} \mathrm{~s}^{-1}$ and $0.16 \mathrm{~km} \mathrm{~s}^{-1}$, respectively. The $\mathrm{NH}_{3}$ and $\mathrm{HC}_{3} \mathrm{~N}$ spectra are shown in Figs. 6 and 7. The hyperfine components show no kinematic substructure and consist of an apparently symmetric peak profile without broadened line wings or self-absorption features. In cores $\mathrm{L} 1512$ and $\mathrm{L} 1498$, the $\mathrm{NH}_{3}$ hyperfine satellite lines with $\Delta F_{1} \neq 0$ are optically thin, $\tau<1$, whereas the main transitions with $\Delta F_{1}=0$ are slightly saturated, $\tau \gtrsim 1$ (Paper II). However, we did not find significant differences between the RVs of the main and satellite lines (see Tables 2 and 3 in Paper II). The optical depth of the strongest hyperfine transition $F^{\prime} \rightarrow F=3 \rightarrow 2$ of $\mathrm{HC}_{3} \mathrm{~N}(2-1)$ is 0.67 (L1498) and 0.35 (L1512).

If the two molecular transitions are thermally broadened and trace the same material, the lighter molecule $\mathrm{NH}_{3}$ should have a wider linewidth than the heavier molecule $\mathrm{HC}_{3} \mathrm{~N}$. This is precisely what is seen in Figs. 6 and 7. Thus the observed spectra from L1512 and L1498 are consistent with thermally dominated line broadening at a kinetic temperature $T_{\text {kin }} \sim 13 \mathrm{~K}$. This may indicate that the two species are sampling the same gas.

Table 2 lists the measured offsets of the $\mathrm{NH}_{3}(1,1)$ and $\mathrm{HC}_{3} \mathrm{~N}(2-1)$ lines as well as their linewidths (observations with $\mathrm{MSpec0}$ ). The measured RVs give a velocity offset $\Delta R V=$ $0.001 \pm 0.009 \mathrm{~km} \mathrm{~s}^{-1}$ for L1512, and $\Delta R V=-0.001 \pm$ $0.012 \mathrm{~km} \mathrm{~s}^{-1}$ for L1498, with the mean $\langle\Delta R V\rangle=0.000 \pm$ $0.009 \mathrm{~km} \mathrm{~s}^{-1}$. The previous observations of L1512 with ARCOS (Paper I) gave the offset $\Delta R V=0.018 \pm 0.012 \mathrm{~km} \mathrm{~s}^{-1}$, which is slightly biased with respect to the current estimate (indicated are $3 \sigma$ C.L.).

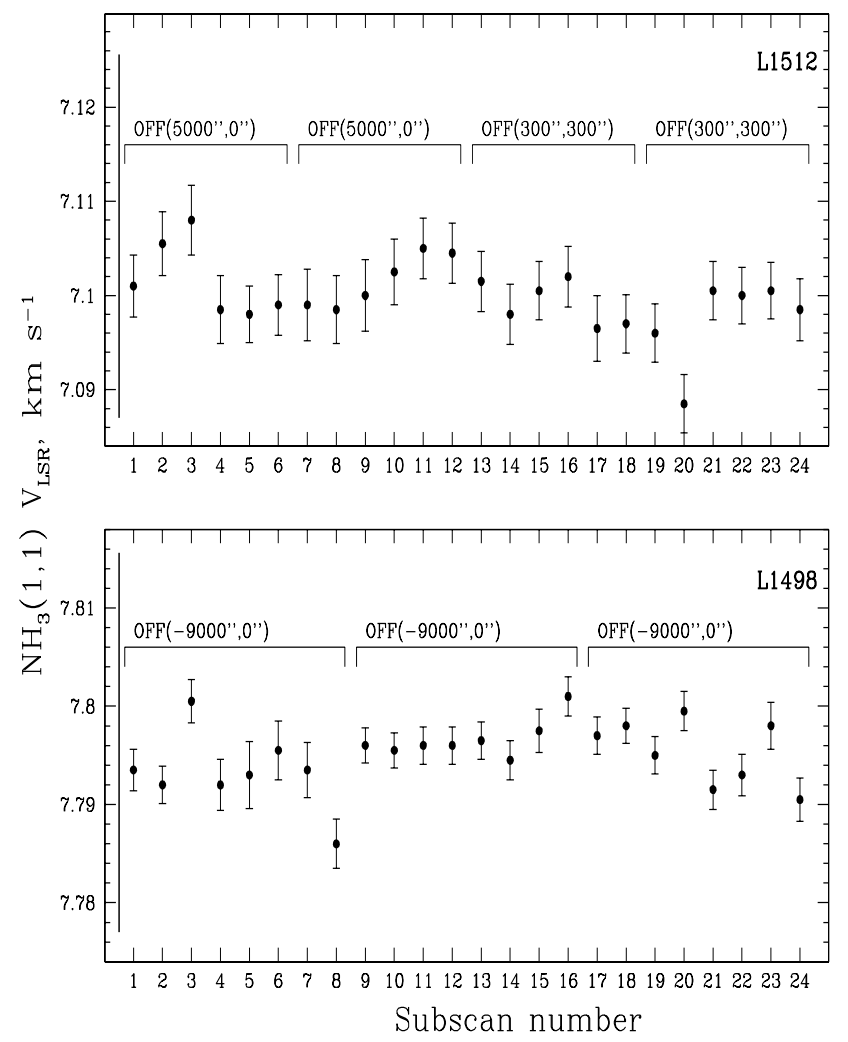

Fig. 4. Line-of-sight velocities $\left(V_{\mathrm{LSR}}\right)$ of the $\mathrm{NH}_{3}(1,1)$ transition (dots with $1 \sigma$ error bars) towards the ammonia peaks in the molecular cores L1512 and L1498 measured continuously in the PSW mode at the Effelsberg 100-m radio telescope in April, 2012. The exposure time at each point is $150 \mathrm{~s}$. The position-switching offsets are shown in parentheses. The backend was an extended fast Fourier-transform spectrometer (XFFTS) with a channel separation $\Delta_{\mathrm{ch}}=0.039 \mathrm{~km} \mathrm{~s}^{-1}$ (marked by vertical lines). Random shifts of the $V_{\mathrm{LSR}}$ values are revealed with an amplitude $\simeq \pm 0.01 \mathrm{~km} \mathrm{~s}^{-1}$ (i.e., $\simeq 1 / 4$ th of the channel width).

Formally, the new observations show an error of the mean $\langle\Delta R V\rangle$ twice lower than that of the Effelsberg result. However, since only one single position was observed in both cores, we cannot evaluate the systematics of MSpec0 (like we do at Effelsberg), and on the basis of the Effelsberg experience we adopt the same limit, $\Delta \mu / \mu<2 \times 10^{-8}$ ( $3 \sigma$ C.L.), that is, we conservatively assign a bias of $\sim 0.02 \mathrm{~km} \mathrm{~s}^{-1}$ to the possible systematic error of the Medicina dataset.

\section{Limits on $\Delta \alpha / \alpha$}

The obtained local constraint on the spatial $\mu$-variation, $\Delta \mu / \mu<$ $2 \times 10^{-8}(3 \sigma$ C.L.), can be used to set limits on changes in $\alpha$. This is strongly model dependent, however. For example, within the grand unification theory (GUT) a variation of $\alpha$ would imply considerably stronger fractional changes in the mass scale of the strong force in $\mathrm{QCD}, \Lambda_{\mathrm{QCD}}$, and in quark- and electron masses, leading to

$\Delta \mu / \mu \sim R \Delta \alpha / \alpha$,

where $R \sim 40$ (e.g., Langacker et al. 2002; Flambaum et al. 2004). This gives a limit on $|\Delta \alpha / \alpha|<10^{-10}$ ( $1 \sigma$ C.L.). A direct estimate of changes in $\alpha$ in the Milky Way, based on observations of $\mathrm{CH}$ sources, sets a $1 \sigma$ upper limit $|\Delta \alpha / \alpha|<10^{-7}$ (Truppe et al. 2013). 


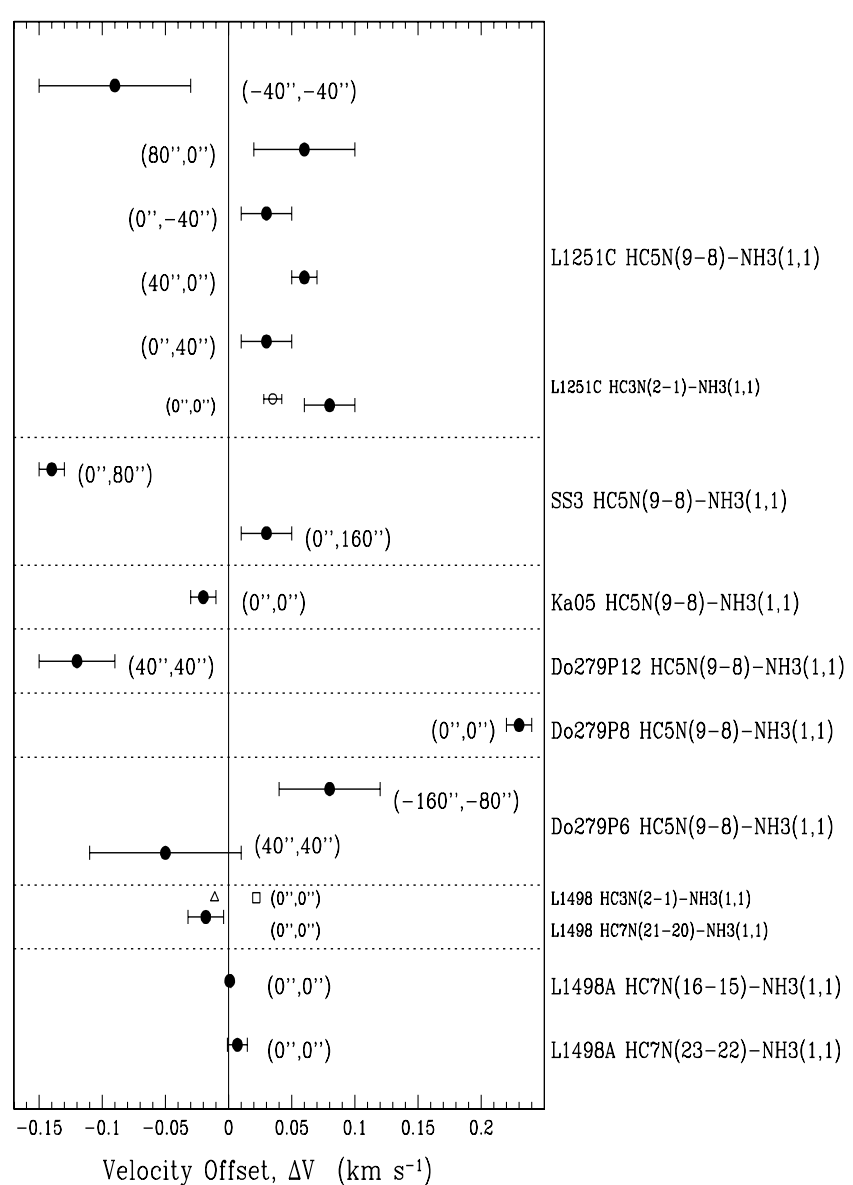

Fig. 5. Radial velocity differences, $\Delta \mathrm{RV}$, between rotational transitions of different molecules and the $\mathrm{NH}_{3}(1,1)$ inversion line for the sources observed at the Effelsberg 100-m telescope (2011-2013). $1 \sigma$ statistical errors are indicated. The coordinate offsets in arcsec are given in parentheses. Filled circles are for data from this paper; open triangle, square, and circle are for data from Papers I and II.

At higher redshifts, the most stringent constraint on cosmological $\mu$-variation was set at $z=0.89,|\Delta \mu / \mu|<10^{-7}$ (Bagdonaite et al. 2013). For $R \sim 40$, this would imply that $|\Delta \alpha / \alpha|<2.5 \times 10^{-9}(1 \sigma$ C.L. $)$ at epoch $7 \times 10^{9}$ yr, meaning in turn that $|\dot{\alpha} / \alpha|<4 \times 10^{-19} \mathrm{yr}^{-1}$. At very high redshift, $z=5.2$ (epoch $12.9 \mathrm{Gyr}$ ), the current limit is $\Delta \alpha / \alpha<8 \times 10^{-6}$ ( $1 \sigma$ C.L.), corresponding to $|\dot{\alpha} / \alpha|<6 \times 10^{-16} \mathrm{yr}^{-1}$ (Levshakov et al. 2012). We recall that most stringent limits set by the Oklo fossil reactor and by terrestrial atomic clock experiments are $|\dot{\alpha} / \alpha|<5 \times 10^{-17} \mathrm{yr}^{-1}$ (Uzan 2011) and $|\dot{\alpha} / \alpha|<4 \times 10^{-17} \mathrm{yr}^{-1}$ (Rosenband et al. 2008), respectively.

As mentioned above, the value of $R$ is poorly constrained. Depending on the theory, it varies from -235 to +46 (see, e.g., Sect. 5.3.1 in Uzan 2011). The only way to distinguish between the theories is to measure $\Delta \alpha / \alpha$ and $\Delta \mu / \mu$ independently.

\section{Conclusions}

We have used the Medicina 32-m telescope and the Effelsberg 100-m telescope to observe the $\mathrm{NH}_{3}(1,1) 23.7 \mathrm{GHz}, \mathrm{HC}_{3} \mathrm{~N}(2-1)$ $18.2 \mathrm{GHz}, \mathrm{HC}_{5} \mathrm{~N}(9-8) 23.9 \mathrm{GHz}, \mathrm{HC}_{7} \mathrm{~N}(16-15) 18.0 \mathrm{GHz}$, $\mathrm{HC}_{7} \mathrm{~N}(21-20) 23.7 \mathrm{GHz}$, and $\mathrm{HC}_{7} \mathrm{~N}(23-22) 25.9 \mathrm{GHz}$ spectral lines in molecular cores devoid of associated IR sources. The principle of local position invariance (LPI) was tested in the solar vicinity ( $D \lesssim 300 \mathrm{pc}$ ) by comparing the electron-to-proton

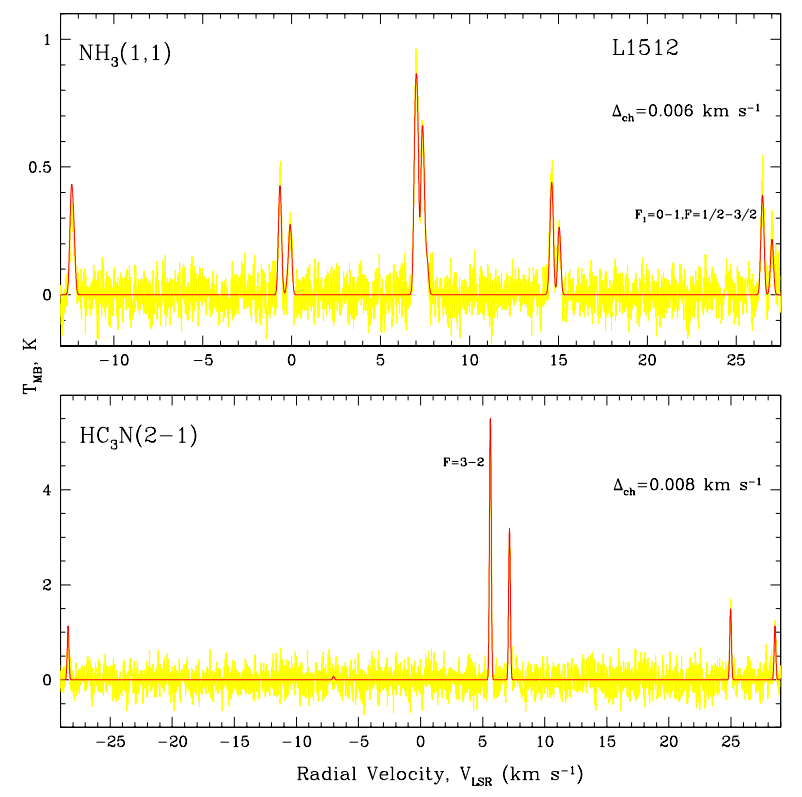

Fig. 6. Spectra of $\mathrm{NH}_{3}(1,1)$ and $\mathrm{HC}_{3} \mathrm{~N}(2-1)$ (yellow) towards core L1512 (offset $\Delta \alpha, \Delta \delta=0^{\prime \prime}, 0^{\prime \prime}$ ) obtained at the Medicina 32-m radio telescope with the high-resolution spectrometer MSpec0 (channel widths $\Delta_{\mathrm{ch}}=0.006 \mathrm{~km} \mathrm{~s}^{-1}$ and $0.008 \mathrm{~km} \mathrm{~s}^{-1}$ at $23 \mathrm{GHz}$ and $18 \mathrm{GHz}$, respectively). The red curves are the best-fit model spectra. The observed linewidths are $\Delta v\left(\mathrm{NH}_{3}\right)=0.19 \mathrm{~km} \mathrm{~s}^{-1}$, and $\Delta v\left(\mathrm{HC}_{3} \mathrm{~N}\right)=0.16 \mathrm{~km} \mathrm{~s}^{-1}$.

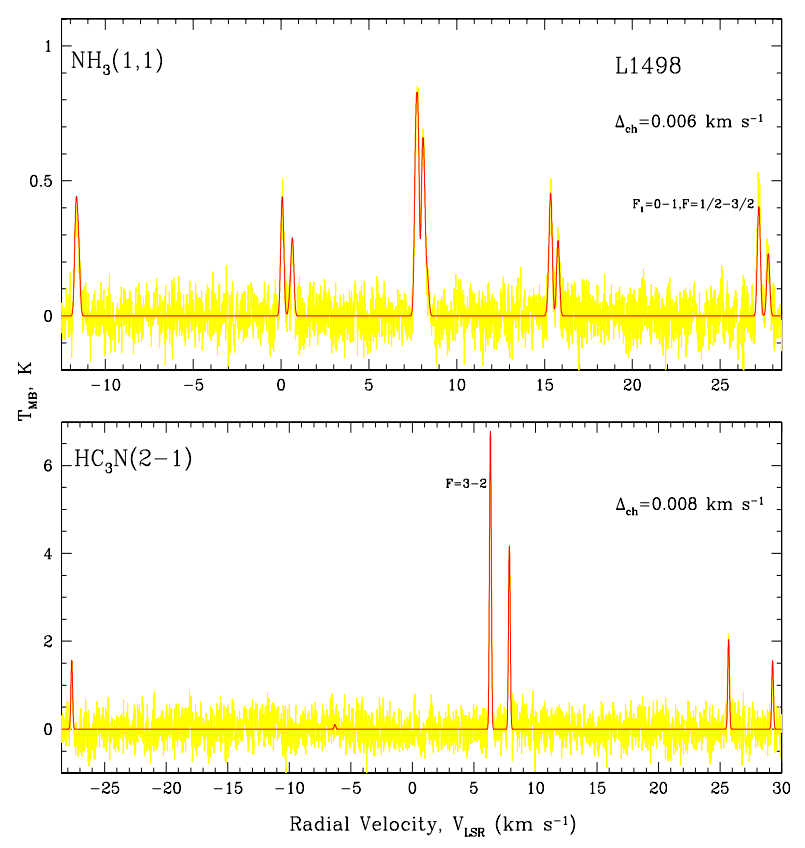

Fig. 7. Same as Fig. 6, but for core L1498. The observed linewidths are $\Delta v\left(\mathrm{NH}_{3}\right)=0.19 \mathrm{~km} \mathrm{~s}^{-1}$, and $\Delta v\left(\mathrm{HC}_{3} \mathrm{~N}\right)=0.16 \mathrm{~km} \mathrm{~s}^{-1}$.

mass ratio $\mu$ in different physical environments of high terrestrial $\left(n \sim 10^{19} \mathrm{~cm}^{-3}\right)$ and low interstellar $\left(n \sim 10^{4} \mathrm{~cm}^{-3}\right)$ densities of baryonic matter. No statistically significant changes in $\mu$ were detected at a level of $\sim 10^{-8}$.

The main results obtained are as follows:

1. To test the reproducibility of the measurements of the relative radial velocities between the $\mathrm{NH}_{3}(1,1)$ and $\mathrm{HC}_{3} \mathrm{~N}(2-1)$ transitions observed towards dark molecular cores in 2008-2010 at the Medicina 32-m and the 
Effelsberg 100-m telescopes, we re-observed two clouds, L1512 and L1498, and revealed discrepancies between the $\Delta \mathrm{RV}=V_{\mathrm{LSR}}\left(\mathrm{HC}_{3} \mathrm{~N}\right)-V_{\mathrm{LSR}}\left(\mathrm{NH}_{3}\right)$ values that are as large as the channel width, $\triangle \mathrm{RV} \lesssim 0.02 \mathrm{~km} \mathrm{~s}^{-1}$.

2. Continuous observations of L1512 and L1498 at Effelsberg in 2012 at a fixed position towards the ammonia peaks showed that the measured radial velocity $V_{\mathrm{LSR}}\left(\mathrm{NH}_{3}\right)$ fluctuates during the exposure time of two hours with an amplitude $\simeq \pm 0.01 \mathrm{~km} \mathrm{~s}^{-1}$, that is, with approximately $1 / 4$ th of the channel width, which does not allow us to measure radial velocities with uncertainties lower than $0.01 \mathrm{~km} \mathrm{~s}^{-1}$.

3. Tests with the synthesizer frequency at $2000.78125 \mathrm{MHz}$ showed that the sky frequency is accurate to about $32 \mathrm{~Hz}$, i.e., $\approx 0.0004 \mathrm{~km} \mathrm{~s}^{-1}$ at the Effelsberg 100 -m telescope.

4. Taking into account the revealed errors and averaging relative velocities over a sample of eight molecular cores observed in 2013, we found a null offset $\langle\Delta R V\rangle=0.003 \pm$ $0.018 \mathrm{~km} \mathrm{~s}^{-1}$ ( $3 \sigma$ C.L.) between the rotational and inversion transitions of the above mentioned molecules observed with the Effelsberg 100-m telescope. If this offset is interpreted in terms of $\Delta \mu / \mu=\left(\mu_{\mathrm{obs}}-\mu_{\mathrm{lab}}\right) / \mu_{\mathrm{lab}}$, the spatial $\mu$-variation is constrained at the level of $\Delta \mu / \mu<2 \times 10^{-8}$ ( $3 \sigma$ C.L.), which is the strictest limit for the validity of the LPI principle based on radio astronomical observations.

5. A similar null offset was found from high spectral resolution observations of the two cores L1512 and L1498 with the Medicina 32-m telescope in 2010: $\langle\Delta R V\rangle=0.000 \pm$ $0.009 \mathrm{~km} \mathrm{~s}^{-1}(3 \sigma$ C.L. $)$.

Acknowledgements. We thank the staff of the Effelsberg 100-m telescope and the Medicina 32-m telescope for the assistance in observations and acknowledge the help of Giuseppe Maccaferri. SAL's work is supported by the grant DFG Sonderforschungsbereich SFB 676 Teilprojekt C4, and in part by Research Program OFN-17 of the Division of Physics, Russian Academy of Sciences.

\section{References}

Agafonova, I. I., Molaro, P., Levshakov, S. A., \& Hou, J. L. 2011, A\&A, 529, A28

Bagdonaite, J., Jansen, P., Henkel, C., et al. 2013, Science, 339, 46
Bahcall, J. N., Sargent, W. L. W., \& Schmidt, M. 1967, ApJ, 149, L11

Barnard, E. E. 1927, A photographic Atlas of selected regions of the Milky Way (Carnegie Inst. Washington D.C. Publ.)

Caselli, P., Benson, P. J., Myers, P. C., \& Tafalla, M. 2002, ApJ, 572, 238

Chand, H., Petitjean, P., Srianand, R., \& Aracil, B. 2005, A\&A, 430, 47

Clemens, D. P., \& Barvainis, R. 1988, ApJS, 68, 257

Cohen, M., \& Kuhi, L. V. 1979, ApJS, 41, 743

Dobashi, K., Uehara, H., Kandori, R., et al. 2005, PASJ, 57, 1

Flambaum, V. V., \& Kozlov, M. G. 2007, Phys. Rev. Lett., 98, 240801

Flambaum, V. V., Leinweber, D. B., Thomas, A. W., \& Young, R. D. 2004, Phys. Rev. D., 69, 115006

Fuller, G. A., \& Myers, P. C. 1993, ApJ, 418, 273

Hotzel, S., Harju, J., \& Walmsley, C. M. 2004, A\&A, 415, 1065

Klein, B., Hochgürtel, S., Krämer, I., et al. 2012, A\&A, 542, L3

Kozlov, M. G., \& Levshakov, S. A. 2013, Ann. Phys., 525, 452

Kostelecký, V. A., Lehnert, R., \& Perry, M. J. 2003, Phys. Rev. D, 68, 123511

Kuiper, T. B. H., Langer, W. D., \& Velusamy, T. 1996, 468, 761

Langacker, P., Segré, G., \& Strassler, M. J. 2002, Phys. Lett. B, 528, 121

Levshakov, S. A. 1992, in High Resolution Spectroscopy with the VLT, ed. M.-H. Ulrich (ESO: Garching/Munchen), 139

Levshakov, S. A., Molaro, P., Lapinov, A. V., et al. 2010a, A\&A, 512, A44 (Paper I)

Levshakov, S. A., Lapinov, A. V., Henkel, C., et al. 2010b, A\&A, 524, A32 (Paper II)

Levshakov, S. A., Combes, F., Boone, F., et al. 2012, A\&A, 540, L9

Levshakov, S. A., Henkel, C., Reimers, D., et al. 2013, A\&A, 553, A58

Liberati, S. 2013, Class. Quant. Grav., 30, 133001

Molaro, P., Centurión, M., Whitmore, J. B., et al. 2013, A\&A, 555, A68

Murphy, M. T., Webb, J. K., Flambaum, V. V., Prochaska, J. X., \& Wolfe, A. M. 2001, MNRAS, 327, 1237

Olano, C. A., Walmsley, C. M., \& Wilson, T. L. 1988, A\&A, 196, 194

Pagani, L., Daniel, F., \& Dubernet, M.-L. 2009, A\&A, 494, 719

Quast, R., Reimers, D., \& Levshakov, S. A. 2004, A\&A, 415, L7

Rosenband, T., Hume, D. B., Schmidt, P. O., et al. 2008, Science, 319, 1808

Savedoff, M. P. 1956, Nature, 178, 688

Srianand, R., Chand, H., Petitjean, P., \& Aracil, B. 2007, Phys. Rev. Lett., 99, 239002

Tafalla, M., Myers, P. C., Caselli, P., \& Walmsley, C. M. 2004, A\&A, 416, 191

Tafalla, M., Santiago-García, J., Myers, P. C., et al. 2006, A\&A, 455, 557

Truppe, S., Hendricks, R. J., Tokunaga, S. K., et al. 2013, Nat. Com., 4, 2600

Uzan, J.-P. 2011, Living Rev. Relativity, 14, 2

Varshalovich, D. A., \& Potekhin, A. Y. 1995, SSRv, 74, 259

Varshalovich, D. A., Panchuk, V. E., \& Ivanchik, A. V. 1996, Astro. Lett., 22, 6 Webb, J. K., Flambaum, V. V., Churchill, C. W., Drinkwater, M. J., \& Barrow, J. D. 1999, Phys. Rev. Lett., 82, 884 